FORMATION Formation emploi

Revue française de sciences sociales

144 | Octobre-Décembre

Le décrochage scolaire à l'aune des inégalités

territoriales: nouvelles perspectives?

\title{
Public, privé et éducation prioritaire : une analyse comparative de la mixité sociale à l'école
}

Public, Private and Priority education zones: a comparative analysis of social mixing at school

Bildung im öffentlichen, privaten und vorrangigen Sektor : eine vergleichende Analyse der sozialen Vielfalt an den Schulen

Lo público, lo privado y la educación prioritaria : un análisis comparativo de la diversidad social en la escuela

Pierre Courtioux et Tristan-Pierre Maury

\section{CpenEdition}

Journals

Édition électronique

URL : http://journals.openedition.org/formationemploi/6703

DOI : 10.4000/formationemploi.6703

ISSN : 2107-0946

Éditeur

La Documentation française

Édition imprimée

Date de publication : 31 décembre 2018

Pagination : 133-154

ISSN : 0759-6340

Référence électronique

Pierre Courtioux et Tristan-Pierre Maury, «Public, privé et éducation prioritaire : une analyse comparative de la mixité sociale à l'école », Formation emploi [En ligne], 144 | Octobre-Décembre, mis en ligne le 31 décembre 2019, consulté le 06 janvier 2021. URL : http://journals.openedition.org/ formationemploi/6703 ; DOI : https://doi.org/10.4000/formationemploi.6703 


\title{
Public, privé et éducation prioritaire : une analyse comparative de la mixité sociale à l'école
}

\author{
Pierre Courtioux \\ Directeur de recherche en économie à I'EDHEC (École des Hautes Études Commerciales), \\ affilié au pôle de recherche en économie
}

Tristan-Pierre Maury Professeur d'économie à l'EDHEC (École des Hautes Études Commerciales), affilié au pôle de recherche en économie

Résumé

Public, privé et éducation prioritaire : une analyse comparative de la mixité sociale à l'école

Sur la base d'indices de ségrégation portant sur la période 2004-2014, nous analysons le degré de mixité sociale des collèges appartenant aux secteurs privé, public hors ZEP (zone d'éducation prioritaire) et ZEP. Le secteur privé est légèrement surreprésenté parmi les établissements les plus "mixtes ", ainsi qu’au sein des plus "ségrégés ". À niveau donné de mixité sociale, le secteur privé accueille relativement moins d'élèves défavorisés. Parmi les établissements les plus "ségrégés ", dans les secteurs privé et public hors ZEP, il existe une tendance à l'entre-soi des élèves "très favorisés ».

Mots clés : enseignement public, enseignement prive, ZEP - zone d'éducation prioritaire, collège, origine sociale, inégalité sociale, politique de l'éducation

Abstract

Public, Private and Priority education zones: a comparative analysis of social mixing at school

Based on social segregation indices for the period 2004-2014, we compare the level of social mixing at the middle school level between private schools and public schools in and out of priority education zones (ZEP). Private schools are slightly over-represented among the most "mixed" as well as the most "segregated" schools. At a given level of diversity, private schools receive relatively less disadvantaged social backgrounds pupils. Excluding ZEP schools, the most "segregated" schools have a tendency to separate students with advantaged social background and other students. 
Keywords: state education, private education, priority education zone, lower secondary school, social origin, social inequality, education policy

Journal of Economic Literature: I 21 ; I 28

Traduction : Auteurs.

Au sein des débats récents sur les inégalités sociales à l'école (cf. les enquêtes PISA analysées dans OCDE 2016, notamment), la mixité sociale à l'école est aujourd'hui un enjeu politique majeur. En effet, en maintenant une partie des élèves d'origine sociale défavorisée dans un environnement moins propice à la réussite scolaire, l'absence de mixité sociale semble remettre en cause le principe "d'égalité des chances " au fondement de l'école républicaine française. Le rapport d'information de Durand \& Salles (2015) détaille le caractère durablement inégalitaire de l'école en France. Ainsi, les différents indicateurs de mixité sociale ne laissent apparaître aucune amélioration depuis vingt ans (Trancart, 2012 ; Thaurel-Richard \& Murat, 2013). De plus, si les effets globaux de la mixité sociale sur la réussite scolaire sont difficiles à mesurer, plusieurs auteurs insistent sur le renforcement de " ghettos scolaires " ${ }^{1}$ et leur effet négatif sur la qualité des apprentissages (Van Zanten \& Obin, 2008).

S'il est clair que l'objectif de mixité sociale est prédominant, se pose la question de la nature des politiques à mettre en ouvre. De ce point de vue, des expérimentations visant à renforcer la mixité sociale dans les collèges ont été menées depuis la rentrée 2016. Elles ont mobilisé différents leviers (mise en place de secteurs multi-collèges, modification de la sectorisation, création de parcours d'excellence, fermeture/ construction de collèges). Ces expérimentations sont mises en œuvre à un niveau local, en mobilisant l'ensemble des acteurs au sein de "territoires pilotes".

S’il est encore trop tôt pour pouvoir apprécier les résultats de ces actions, il est intéressant de noter quelques éléments concernant leur réception au niveau territorial. En effet, dans certaines situations ${ }^{2}$, des acteurs locaux (familles et enseignants) ont remis en cause le " choix (présenté comme) naturel " de ces territoires, en fustigeant "l'absence d'une vision globale ", qui conduisait ces politiques à "reporter sur un nombre limité de familles la charge à réaliser, voire de compenser l'échec de véritables politiques publiques de mixité à l'échelle de l'académie ". De ce point de vue, la contestation de la légitimité de l'action publique territorialisée s'appuyait sur la contestation des frontières du territoire devant servir de référence. Ainsi, les partisans de l'expérimentation mettaient en avant la place relativement favorisée en termes de mixité sociale du collège sujet de l'expérimentation, tandis que le collectif de parents et de professeurs contestait ce diagnostic en étendant la comparaison à l'ensemble des collèges de l'académie et/ou en

1. L’emploi de ce qualificatif est détaillé et justifié par Durand \& Salles (2015).

2. Notamment dans le cas du collège Coysevox du 18e arrondissement de Paris, cf. Collectif Coysevox, 2016. 
comparant avec la situation relative, en termes de mixité sociale, de collèges jouxtant la zone d'expérimentation.

Indépendamment de l'analyse sociologique que l'on peut réaliser sur ces mobilisations d'acteurs ${ }^{3}$, rares sont les travaux permettant de décrire l'hétérogénéité des collèges en termes de mixité sociale sur l'ensemble du territoire. De plus, les éléments disponibles sur cette hétérogénéité sont d'autant plus rares que l'on s'intéresse aux catégorisations habituelles des politiques éducatives : l'éducation prioritaire et le secteur privé.

Par exemple, les récents travaux de Givord \& al. (2016a, 2016b) opposent bien le public et le privé au niveau national, mais ils ne cherchent pas, au sein du public, à distinguer les collèges relevant de l'éducation prioritaire, dont on s'attend a priori à ce qu'ils soient peu mixtes socialement, du reste du secteur public, pour lequel il serait intéressant de savoir s'il est proche ou non du secteur privé en termes de mixité sociale. Par ailleurs, certains travaux, comme ceux d'Audren \& Lorcerie (2013), ont déjà montré qu'au sein d'un même territoire, il existait des différences très importantes entre collèges privés quant à l'accueil de public divers.

Si les travaux de Givord \& al. (op. cit.) confirment cette forte hétérogénéité du secteur privé au niveau national, ils n'en éclairent pas l'origine : les collèges privés très mixtes socialement sont-ils comparables aux collèges publics ne relevant pas de l'éducation prioritaire ? L'absence de mixité dans un collège privé renvoie-t-elle systématiquement à une stratégie «d'entre-soi » des plus favorisés?

Il est dès lors nécessaire de définir la mixité sociale et sa mesure. Notre travail s’inscrit dans le prolongement de travaux français disponibles sur le sujet ${ }^{4}$. C'est pourquoi nous nous sommes appuyés sur des indices de mesure de la mixité sociale ou de ségrégation déjà utilisés dans le débat français ${ }^{5}$ : les indices d'entropie et d'exposition normalisés. De même, nous retenons une classification de la diversité sociale en quatre catégories (très favorisés, favorisés, intermédiaires et défavorisés), déjà utilisée dans les travaux récents (cf. encadré 2 ).

L'originalité de notre approche consiste principalement à combiner ces deux indices pour caractériser chacun des collèges. L'indice d'entropie nous permet de savoir dans quelle mesure les collèges sont " mixtes ", c’est-à-dire mélangent les quatre catégories correspondant à notre définition de la diversité sociale. Quant à l'indice d'exposition

3. Dans une perspective de sociologie des organisations, il est possible d'interpréter ces éléments de contestation des politiques publiques comme des stratégies d'acteurs éclairant la dynamique et les limites de la gouvernance éducative locale ; Van Zanten \& da Costa (2013) en fournissent, par exemple, une analyse dans le cadre de la réforme de 2007 visant à assouplir la carte scolaire.

4. Notamment Givord \& al. (op. cit.).

5. De ce point de vue, la mixité sociale est mesurée par une famille d'indices statistiques, souvent appelés indices de ségrégation ; c'est pourquoi nous emploierons indifféremment les deux termes "ségrégation " et " mixité " pour caractériser le même phénomène dans la suite du texte. 
normalisé aux très favorisés, il nous permet, à niveau de mixité donné, de préciser dans quelle mesure les très favorisés restent entre eux dans chaque collège ${ }^{6}$.

Dans ce cadre, l'objectif de cet article est bien de compléter les résultats disponibles au niveau national, dont on a vu qu'ils constituaient un élément difficile à écarter dans les débats entre les acteurs parti-prenantes des politiques publiques visant à favoriser la mixité sociale, en prenant soin : i) de distinguer, au sein du secteur public, deux catégories de collèges dont on s'attend à ce qu'ils soient très différents en termes de mixité sociale (les collèges relevant de l'éducation prioritaire et les autres), ii) et d'identifier, au sein du secteur privé, mais également au sein du secteur public, les collèges relevant d'une stratégie " d'entre-soi » des plus favorisés.

Cette démarche nous semble pertinente pour éclairer les enjeux de politique d'éducation au niveau national. En effet, face à une qualité de l'offre éducative très variable sur le territoire, aux stratégies d'évitement des mauvais établissements de la part des familles, ainsi qu’à la liberté de recrutement dont bénéficie le secteur privé, un certain nombre d'experts recommandent l'imposition d'un système de quotas de recrutement, selon l'origine sociale ou scolaire de l'élève, au secteur public et aux collèges privés? Ils défendent ainsi l'idée selon laquelle le secteur de l'établissement représente un filtre pertinent pour les politiques éducatives. Ces recommandations ne prennent donc pas en compte l'hétérogénéité intra-sectorielle que nous mettons ici en évidence.

Pour éclairer cette question, dans une première partie, nous précisons les débats actuels concernant la place du privé et celle des dispositifs d'éducation prioritaire, en France. Nous présentons, ensuite, les deux principaux outils de mesure de la mixité sociale (les indices d'entropie et d'exposition normalisés). Dans une seconde partie, nous caractérisons d'abord les niveaux de mixité sociale à l'aide de l'indice d'entropie, puis nous commentons l'apport d'une analyse combinant l'indice d'entropie et l'indice d'exposition normalisé aux très favorisés.

\section{Les enjeux de la mixité sociale selon le secteur privé, public ou d'éducation prioritaire}

Nous commençons par rappeler le contexte institutionnel français et les caractéristiques générales du secteur public, du secteur privé et de l'éducation prioritaire ; puis nous expliquons comment les indices de ségrégation permettent d'éclairer ces enjeux de mixité sociale et de dégager un certain nombre de faits stylisés sur l'éducation secondaire en France. Les deux principaux faits stylisés nouveaux que nous dégageons sont : tout

6. Cf. infra, partie 1.2 pour une illustration.

7. Comme ont pu le suggérer Maguain (2009), Fack \& Grenet (2009) ou Merle (2010). 
d'abord, une légère surreprésentation du secteur privé parmi les collèges les plus mixtes et parmi les collèges les moins mixtes, enfin, au sein des collèges les moins mixtes, une tendance à la baisse de "l'entre soi » des élèves très favorisés plus forte dans le privé que dans le public hors éducation prioritaire sur la période 2004-2014.

\subsection{Secteurs public, privé et de l'éducation prioritaire}

En France, le secteur privé a un statut bien particulier. Depuis la loi Debré de 1959, ce secteur, issu principalement de l'enseignement confessionnel catholique, est devenu progressivement l'une des composantes du service public d'éducation français (Poucet, 2012 ; Thélot, 2013). En échange de cette participation à un service public d'éducation dont les modalités sont définies par un contrat, il bénéficie de subventions importantes (notamment le financement du salaire des enseignants et de certains frais de fonctionnement). Cependant, le secteur privé n'est pas gratuit et « les classes sociales les plus favorisées y occupent une place majoritaire ${ }^{8}$.

Les différences de recrutement selon l'origine sociale entre public et privé, associées à l'importance des subventions publiques au secteur privé, ont conduit un certain nombre d'experts à remettre en cause la liberté de recrutement des élèves dans le secteur privé (quotas de bons et mauvais élèves, cf. Maguain (op. cit.)ou quotas de recrutement selon l'origine sociale, cf. Fack \& Grenet (op. cit.) ou Merle (op. cit.)). On dispose certes de quelques études de cas, notamment dans la région parisienne, qui indiquent que le secteur privé a eu tendance à diminuer sa diversité de recrutement ces dernières années ${ }^{9}$ et de quelques éléments généraux sur une segmentation sociale entre public et privé plus marquée au cours du temps (Givord \& al., 2016b). Cependant, on sait également que les établissements privés sont très hétérogènes, notamment car ils se caractérisent par une autonomie de gestion au niveau de l'établissement.

La politique de zones prioritaires et les programmes d'éducation prioritaire ont été créés en 1981. Cette politique visait à "renforcer par des moyens sélectifs groupés en programmes d'éducation prioritaires, l'action éducative dans les zones où se concentrent les plus grandes difficultés sociales ${ }^{10}$. L'objectif de ce dispositif était de lutter contre les inégalités, notamment sociales, devant l'école. Elle répondait également à la préoccupation d' "accroître l'égalité des chances offertes aux jeunes scolarisés dans les établissements publics " (Radica, 1995). Il s'agissait d'une expérience, alors unique en France, de territorialisation d'une politique publique : contribuer à l'égalité des chances des élèves via une répartition inégalitaire des moyens mis en œuvre sur le territoire national. Chaque zone prioritaire ZEP a été définie par arrêté préfectoral sur la base de recommandations des recteurs d'académie ; ceux-ci

8. Cf. Poucet (op. cit.), p. 114 ou Prost (2013) qui analysent ces différences dans une perspective historique ou Courtioux (2016), dans une perspective statistique.

9. Par exemple, Merle (op. cit.).

10. Circulaire du ministère de l'Éducation nationale n81-536, du 28 décembre 1981. 
ont délimité des zones où la proportion d'élèves issus de milieux "défavorisés ${ }^{11}$ " était importante.

Par la suite, la carte des ZEP a été redéfinie (en 1989/1990 et 1994 notamment) et a connu plusieurs relances majeures (en 1997, avec la création des Réseaux d'éducation prioritaires - REP ; en 2006, avec la mise en place des Réseaux ambition réussite - RAR et en 2011, avec le programme Écoles, collèges, lycées pour l'ambition, l'innovation et la réussite - ÉCLAIR).

\subsection{Mesurer la mixité sociale à l'aide d'indicateurs statistiques de ségrégation}

Récemment, plusieurs contributions ont proposé d'utiliser des indicateurs de ségrégation pour mesurer la mixité sociale dans le système éducatif secondaire en France ; on peut notamment citer Ly \& al. (2014), Ly \& Riegert (2014) ou Givord \& al. (2016a). Le principe d'un indice de ségrégation est d'évaluer à quel point une variable d'intérêt peut avoir, à l'échelon local, une distribution différente de celle observée sur la population totale. Par exemple, dans le milieu scolaire, cela permet de mesurer l'écart entre la répartition des élèves par origine sociale dans les différents collèges et la répartition nationale. Un indice de ségrégation ne permet donc pas de porter une quelconque appréciation sur la situation globale, mais uniquement sur des écarts locaux à cette situation globale.

De nombreux indices existent pour aborder ces questions (voir encadré 1), mais deux d'entre eux sont plus fréquemment utilisés : l'indice d'entropie normalisé et l'indice d'exposition normalisé. Ces indices partagent des propriétés statistiques appréciables. Ils sont notamment tous deux décomposables ${ }^{12}$, ce qui, dans le cadre de notre recherche, permet d'isoler les contributions de chaque secteur : privé, public hors ZEP et ZEP. Pour les calculer, nous utilisons une classification sociale en quatre groupes : très favorisés, favorisés, intermédiaires et défavorisés (encadré 2).

11. Selon des critères reliés à la catégorie socio-professionelle, la nationalité ou le niveau éducatif des parents ou à la scolarité des enfants.

12. Cf. le point 2 de l'encadré 1 pour plus de précisions. 


\section{Encadré 1. Les indices de ségrégation}

Il existe de nombreux indices de ségrégation (à titre d'exemple, Massey \& Denton, 1988, en recensent plus d'une vingtaine). Récemment, Reardon \& Firebaugh (2002) \& Frankel \& Volij (2011) ont effectué une analyse comparative détaillée des propriétés des indices les plus fréquemment utilisés. Parmi ces propriétés, deux sont particulièrement importantes :

1- L'invariance à la composition établit que si le nombre d'individus d'une catégorie donnée (les très favorisés, par exemple) venait à augmenter, mais que ces nouveaux individus sont répartis dans les collèges selon la répartition nationale existante, alors l'indice de ségrégation ne doit pas changer. L'invariance à la composition permet donc d'effectuer des comparaisons géographiques ou temporelles : les niveaux de ségrégation de deux régions différentes (ou pour deux années académiques différentes) peuvent être comparés, même si leurs compositions (part de très favorisés, de défavorisés, etc.) ne sont pas les mêmes ;

2- La décomposition qui établit que, après avoir effectué des regroupements, l'indice est décomposable en une somme de composantes intra-groupes et inter-groupes indépendantes. Par exemple, dans cet article, cela permet de décomposer le niveau de ségrégation en une composante intersectorielle (hétérogénéité entre les trois secteurs, privé, public et ZEP - zone d'éducation prioritaire) et une composante intra-sectorielle (hétérogénéité au sein des trois secteurs).

Sur la base de ces deux propriétés, quatre indices peuvent être retenus (cf. Frankel \& Volij, op. cit.) : les indices d'exposition et d'entropie normalisés (que nous utilisons), l'indice d'Atkinson et l'indice d'information mutuelle. L'indice d'Atkinson, proposé par James \& Taeuber (1985), présente l'avantage de vérifier l'invariance à la composition (à la différence des trois autres indices), mais est peu interprétable et faiblement décomposable. II faut noter que, même si les indices d'exposition et d'entropie normalisés ne sont pas, stricto sensu, invariants à la composition, certaines études empiriques ont montré qu'ils variaient relativement peu selon la composition (cf. Reardon \& O'Sullivan, 2004).

L'indice d'information mutuelle est similaire à l'indice d'entropie, mais n'est pas normalisé. Cela rend cet indice "fortement » décomposable, c'est-à-dire que les poids de la décomposition sont nationaux (taille du public, taille du privé) et ne dépendent pas de la répartition des élèves au sein de chaque secteur (ex : répartition des très favorisés, des favorisés, etc., au sein du secteur privé). L'indice d'entropie normalisé n'est que « faiblement » décomposable : les poids dépendent de la répartition des élèves au sein de chaque groupe. En dépit de ses qualités, mais du fait de sa non-normalisation, l'indice d'information mutuelle est difficile à interpréter et n'est donc pas retenu.

L'indice d'exposition normalisé, tel qu'utilisé dans cet article, est fortement décomposable. Nous travaillons donc avec les indices d'exposition et d'entropie normalisés, tous deux décomposables - fortement ou faiblement - peu sensibles à la composition et facilement interprétables. De plus, par ce choix, nos résultats peuvent être comparés à ceux de Givord \& al. (2016a) et Ly \& al. (op. cit.) qui, dans un contexte français, utilisent ces mêmes indices. 
Avec l'indice d'entropie normalisé $(H)$, la mixité sociale au niveau d'un collège se mesure par la distance entre la diversité sociale au sein de ce collège et dans une population de référence, ici composée de l'ensemble des collèges français (encadré 2). Il ne s'agit donc pas d'un indice de ségrégation qui chercherait, par exemple, à identifier les collèges avec une forte polarisation de défavorisés ou au contraire une forte polarisation de très favorisés. Avec cette mesure, un collège est d'autant plus mixte que sa composition sociale, dans toutes ses dimensions, se rapproche de la composition sociale de la population de référence.

L'indice $H$ est conçu comme la somme de l'éloignement de la composition sociale de chaque collège à la composition sociale de l'ensemble de la population de référence. L'indice d'entropie est compris entre 0 et 1 . Plus cet indice est élevé, plus il indique une dissimilarité entre les collèges. Il vaut 1 lorsqu'aucune mixité n'est observée (chaque collège n'est constitué que d'un groupe social). Au contraire, plus il est proche de 0 , plus il indique une homogénéité de composition entre les différents collèges.

Dans ce cadre, il est également possible de calculer ces indices de mixité sociale en distinguant différents secteurs. Nous distinguons ainsi trois secteurs : le privé, le public en "ZEP» et le public autre. En utilisant les propriétés statistiques de l'indice d'entropie $H$, nous pouvons le décomposer en quatre éléments : (1) l'indice d'entropie au sein des collèges privés, (2) l'indice d'entropie au sein du public hors ZEP, (3) l'indice d'entropie au sein des ZEP et (4) l'indice d'entropie lié à la segmentation intersectorielle (c'est-à-dire entre ZEP, public hors ZEP et privé, chacun considéré comme un seul grand établissement).

Si l'indice d'entropie est un outil souple et utile pour mesurer la mixité sociale, il ne la résume cependant pas. En effet, il y a de nombreuses manières de "produire " de l'entropie. Ainsi, si l'on compare deux établissements, l'un comportant une moitié d'élèves issus de groupes sociaux " très favorisés » et une moitié d'élèves issus de groupes sociaux "défavorisés ", et l'autre collège comportant une moitié d'élèves issus de groupes sociaux "favorisés " et une moitié d'élèves issus de groupes sociaux " moyens ", ces deux établissements ont exactement le même niveau d'entropie. On peut cependant considérer a priori que leur contribution à la mixité sociale n'est pas la même : un seul des deux établissements fait cohabiter des élèves issus d'origines sociales très éloignées.

Nous utilisons donc un autre indicateur de ségrégation sociale : l'indice d'exposition normalisé $(P)$ qui vient compléter l'indice d'entropie. L'indice d'exposition est moins général et se focalise sur un des groupes sociaux : le groupe de référence. A la différence de l'indice d'entropie, aucune distinction ne sera faite entre les autres groupes : seule l'appartenance - ou non - au groupe de référence est déterminante. L'indice $P$ peut s'écrire comme suit :

$$
P=\mu_{1}-\mu_{0}
$$

où $\mu_{1}$ est le taux d'exposition au groupe de référence (calculé à l'échelon du collège) pour les élèves eux-mêmes issus du groupe de référence. Si l'on considère que le groupe de référence est constitué des élèves " très favorisés ", $\mu_{1}$ est obtenu en estimant la 
moyenne de la part d'élèves très favorisés dans le collège de chaque élève très favorisé. $\mu_{0}$ est le taux d'exposition au groupe de référence pour les élèves issus des autres groupes. Avec l'exemple précédent, il s'agit de la moyenne de la part d'élèves très favorisés présents dans leur collège pour les élèves des groupes favorisés, intermédiaires et défavorisés.

$P$ est compris entre 0 et 1 . Si l'indice $P$ est égal à 0 , cela signifie que la répartition des élèves est la même dans tous les collèges : l'exposition au groupe de référence est la même partout, la mixité est donc maximale. Au contraire, si $P$ est égal à 1 , la ségrégation est totale : les élèves du groupe de référence ne sont exposés qu'à leur propre groupe $\left(\mu_{1}=1\right)$ et les élèves d'autres groupes ne sont jamais exposés à ceux du groupe de référence $\left(\mu_{0}=0\right)$. Dans ce cas, il n'y a que deux types de collèges : ceux constitués exclusivement d'élèves du groupe de référence et ceux constitués sans aucun élève du groupe de référence.

Les deux indices de ségrégation sociale, l'indice d'entropie $(H)$ et d'exposition normalisé $(P)$, fournissent donc des informations qui ne se recoupent qu'en partie. Ils sont bien sûr corrélés : par exemple, si tous les collèges ont la même répartition, alors $H$ sera nul (l'entropie est la même pour tous les collèges) et $P$ aussi (tous les élèves ont la même exposition au groupe de référence). En revanche, dans certaines situations, $P$ et $H$ peuvent diverger, car l'indice d'exposition normalisé ne se concentre que sur un groupe particulier et n'est pas sensible à la répartition des autres groupes. Ainsi, le niveau d'entropie peut être le même pour des collèges très différents : on peut obtenir la même contribution à l'indice d'entropie national pour un collège avec, par exemple, une proportion "normale " de très favorisés, peu de catégories moyennes et beaucoup de catégories défavorisées et un autre collège avec beaucoup de très favorisés, peu de catégories moyennes et une proportion "normale " de défavorisés. Pourtant, ces deux collèges n'ont pas la même exposition aux très favorisés (l'une est conforme à la moyenne nationale et l'autre très forte). Ils n'auront donc pas la même contribution à l'indice d'exposition normalisé calculé au niveau national. Si l'on considère que l'exposition à la catégorie très favorisée est également un élément important de l'analyse, il apparait nécessaire de combiner les indices normalisés d'entropie et d'exposition. 


\section{Encadré 2. Données et variables prises en compte}

Nous utilisons des données issues du système d'information SCOLARITÉ et transmises par le réseau français des centres de données pour les sciences sociales (réseau Quetelet). Les données transmises au réseau Quetelet sont issues de la Base Centrale Scolarité (BCS), qui est elle-même une extraction d'autres bases « fondamentales » plus générales (la Base Élèves Académique, BEA, et la Base Élèves Établissement, BEE).

Nous disposons, pour toutes les années de 2004 à 2014, d'un fichier contenant des caractéristiques administratives et géographiques de l'ensemble des collèges en France (" fichier établissements »). Notamment, nous connaissons le secteur (public/privé), son appartenance éventuelle à un dispositif d'éducation prioritaire et sa localisation (numéro INSEE de la commune). Bien que les Zones d'éducation prioritaires (ZEP) ne soient plus en place depuis 2010, le terme de ZEP est couramment utilisé pour qualifier l'ensemble des politiques d'éducation prioritaire. Nous en retenons ici une acception large qui inclut l'ensemble des dispositifs utilisés sur la période 2004-2014, c'est-à-dire : les ZEP, les Réseaux ambition réussite (RAR), les Réseaux d'éducation prioritaire (REP et REP+), les Réseaux de réussite scolaire (RRS) et le dispositif Écoles, collèges, lycées pour l'ambition, l'innovation et la réussite (ÉCLAIR).

Sur la période que nous étudions, la part d'élèves scolarisés dans le secteur privé a légèrement augmenté entre 2004 et 2014, passant de $20 \%$ à $21 \%$, de même que la part des élèves scolarisés dans des établissements publics hors politique d'éducation prioritaire, qui s'élève à $63 \%$ des élèves en 2004 et $64 \%$ en 2014. Seule la part des élèves scolarisés en ZEP a diminué sur la période, passant de 17 à $16 \%$.

Un « fichier élèves » fournit des caractéristiques socio-démographiques pour chaque individu de la population totale des élèves dans le second degré : sexe, nationalité, origine sociale et département de résidence, ainsi que des éléments sur sa scolarité (formation suivie, langues vivantes étudiées, etc.). Un identifiant de l'établissement permet de faire le lien entre les fichiers « établissements » et " élèves ». À titre d'exemple, pour l'année 2004, nous disposons d'informations individuelles relatives à 3252380 élèves répartis sur 6924 collèges. Pour cette étude, nous n'avons retenu que les collèges présents sur l'ensemble de la période 2004-2014, ce qui ramène le nombre de collèges de notre échantillon à 6722.

Nous utilisons l'origine sociale du responsable de l'élève pour reconstituer la classification des catégories socio-professionnelles (PCS) utilisée par la Direction de l'évaluation, de la prospective et de la performance (DEPP) $\left(^{*}\right)$ : les élèves sont regroupés en quatre groupes sociaux : « très favorisés », " favorisés », « intermédiaires » et « défavorisés ». Bien évidemment, pour un même nombre de catégories, les choix de regroupement peuvent influencer le résultat. Dans ce travail, nous avons décidé d'écarter de l'analyse les élèves pour lesquels la catégorie sociale des parents n'est pas renseignée (**). Il convient également de noter qu'en suivant cette définition, l'origine sociale des élèves a connu des changements sur la période. Les changements les plus forts concernent la part de favorisés et de très favorisés. Entre 2004 et 2014, la part des élèves très favorisés a augmenté de trois points de pourcentage pour atteindre $23 \%$, alors que la part de favorisés quant à elle diminuait de plus de deux points de pourcentage pour se fixer à $6 \%$. La part des élèves d'origine intermédiaire a très légèrement crû (un point de pourcentage environ) pour atteindre $35 \%$ sur la période, alors que la part des défavorisés décroissait de moins d'un point de pourcentage, pour atteindre $36 \%$.

$\left(^{*}\right)$ : Cf. notamment Durand \& Salles (op. cit.), annexe 2, p. 220.

$\left.{ }^{* *}\right)$ : Pour tous les résultats présentés ici, nous avons testé un autre regroupement de PCS inspiré de Courtioux (2016). Ces résultats confirment ceux présentés ici et sont disponibles sur demande auprès des auteurs. 


\section{Le privé, surreprésenté parmi les collèges les plus « mixtes » et les plus " ségrégés "}

Cette partie s'articule en trois sous-parties. Nous étudions d'abord l'évolution de la mixité sociale, telle que mesurée par les indices d'entropie et d'exposition normalisés, entre 2004 et 2014. Ainsi, en termes de mixité sociale, le secteur public ne peut pas être analysé comme un seul bloc, tant les collèges relevant de l'éducation prioritaire sont concentrés au sein des établissements les moins mélangés socialement.

Nos résultats montrent un degré de mixité sociale plutôt stable sur la période. Par ailleurs, il apparait que les collèges privés peuvent être très différents en termes de mixité sociale ; Puis, nous effectuons une décomposition par centile des niveaux d'entropie par collège et comparons la place relative de chaque secteur (public hors ZEP, ZEP et privé) selon le niveau de mixité sociale. Le secteur privé apparait légèrement surreprésenté au sein des collèges les plus mixtes et au sein des collèges les moins mixtes socialement.

Enfin, nous illustrons les différentes manières de "produire " de la mixité sociale. Pour cela, nous comparons les indices d'exposition normalisés de collèges de secteurs différents (public hors ZEP, en ZEP, privé) ayant des niveaux d'entropie comparables. Nous montrons que sur la période, au sein des collèges les moins mixtes, "l'entre soi » des élèves les plus favorisés a diminué plus fortement dans le secteur privé que dans le secteur public hors éducation prioritaire.

\subsection{Un degré de mixité sociale plutôt stable sur la période 2004-2014}

La figure 1 caractérise la segmentation publique hors ZEP/ZEP/privé. La courbe noire en trait plein montre l'évolution de l'indice d'entropie normalisé calculé sur l'ensemble des collèges pour chaque année entre 2004 et 2014. Le niveau d'entropie des différents secteurs de l'enseignement donne la mesure dans laquelle, au sein d'un même secteur, les collèges qui le composent se ressemblent en termes de mixité sociale.

On remarque que les collèges du secteur privé sont les plus hétérogènes. Les collèges publics en ZEP ou hors ZEP se ressemblent plus ; leur indice d'entropie normalisé est moins élevé, c'est-à-dire environ 0,06-0,07, alors qu'il est de l'ordre de 0,11 pour le privé ${ }^{13}$.

La mixité sociale a peu évolué en dix ans. Ce constat est globalement vrai pour tous les secteurs, même si on remarque que les collèges publics n'appartenant pas à l'éducation prioritaire ont une légère tendance au rapprochement de leur composition sociale : ils se ressemblent plus.

13. Notons que quand on rassemble les différents segments du secteur public, c'est-à-dire les collèges en ZEP et les autres, les collèges publics ont tendance à moins se ressembler, mais se ressemblent toujours plus entre eux que les collèges du secteur privé : le niveau de l'indice d'entropie est alors d'environ 0,08. 
Notre résultat peut être interprété comme un léger renforcement de la segmentation sociale entre le secteur public hors ZEP, le secteur ZEP et le secteur privé. En effet, l'indice d'entropie lié à la segmentation entre les trois secteurs d'éducation a légèrement progressé sur la période. Ceci confirme, dans le cadre d'une répartition tri-sectorielle, les résultats de Givord \& al. (2016b) obtenus sur la base d'une opposition public/privé.

L'ensemble de ces résultats indique que faire un état des lieux de la mixité sociale dans les collèges français nécessite d'examiner la disparité au sein de ces différents segments et plus particulièrement au sein du privé, où elle apparaît particulièrement élevée ; ce dernier semblant inclure à la fois des collèges mixtes et des collèges peu mixtes.

Figure 1. Évolution de l'indice d'entropie et de ses composantes

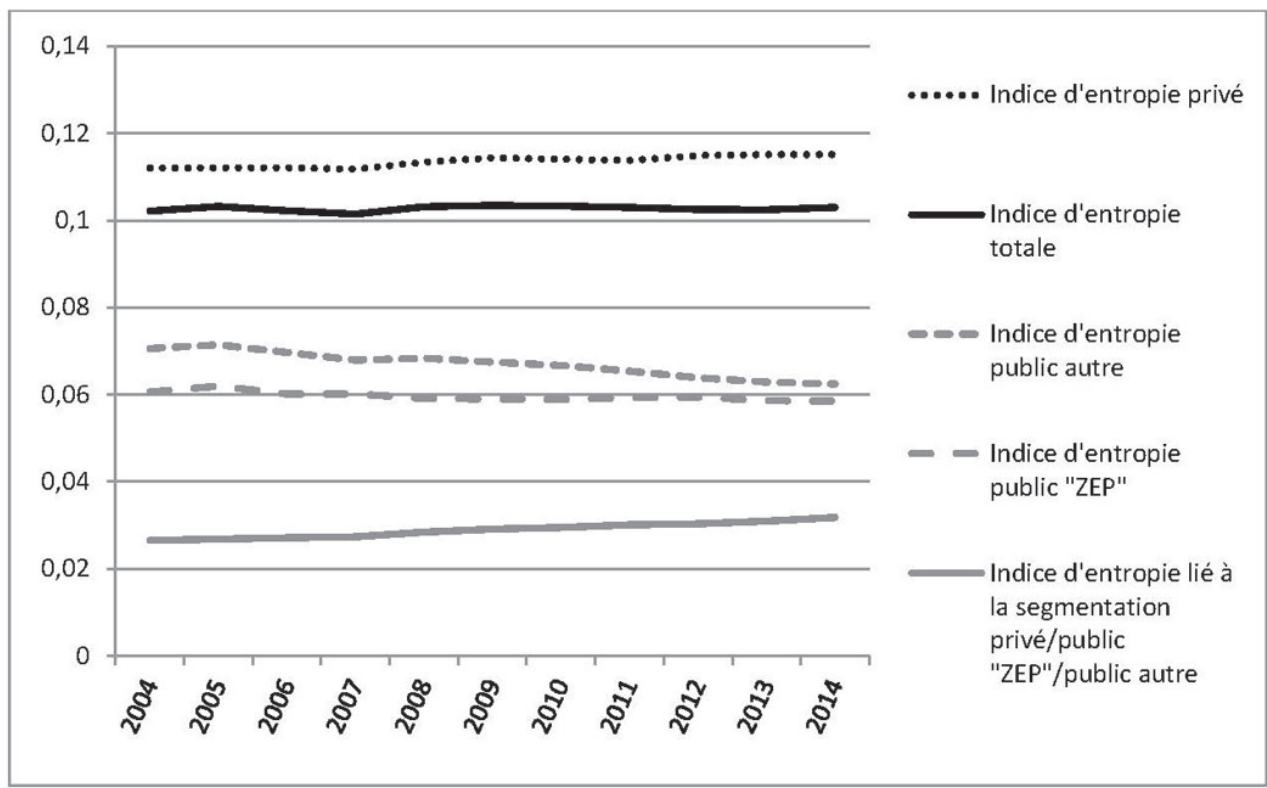

Note de lecture : le niveau de ségrégation sociale pour l'ensemble des collèges mesuré par l'indice d'entropie totale était légèrement supérieur à 0,1 en 2004 et est resté stable entre 2004 et 2014.

Source : Base Centrale de Scolarité (2004-2014) DEPP - calcul des auteurs.

Les résultats fournis par l'indice d'exposition normalisé à la catégorie " très favorisée ", sur la période 2004-2014, sont présentés dans la figure 2.

Cet indice est resté stable sur la période : il était de 15\% en 2004 et de $16 \%$ en 2014, ce qui traduit une très légère augmentation de la segmentation sociale entre collèges sur la période considérée. Ce résultat est cohérent avec celui obtenu avec l'indice d'entropie normalisé (figure 1). Nous pouvons distinguer, au sein de l'indice d'exposition 
normalisé global, la part de cette exposition due à des différences intersectorielles de la part due à des différences intra-sectorielles (c'est-à-dire entre collèges d'un même secteur) : cette part est relativement stable entre 2004 et 2014.

Figure 2. Décomposition de l'indice d'exposition normalisé $\mathrm{P}$ par année.

Catégorie de référence : élèves très favorisés.

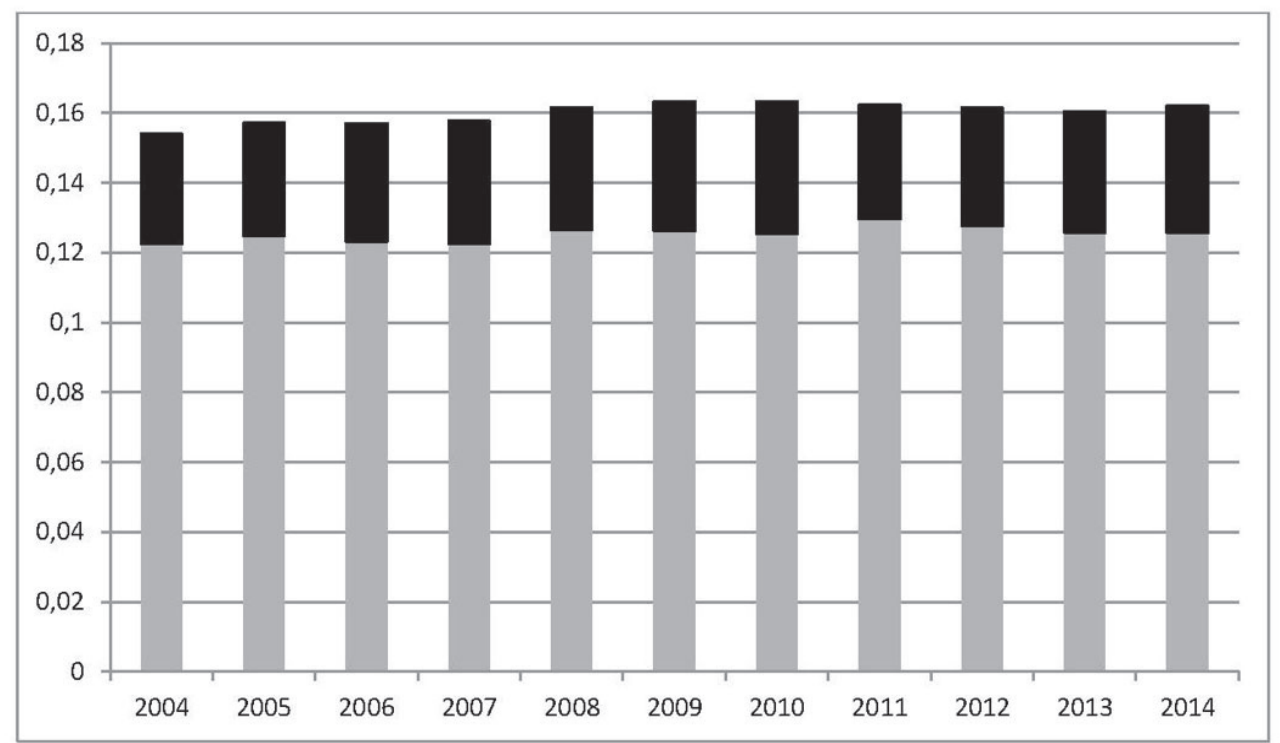

Lecture : Décomposition de P entre collèges d'un même secteur pour le gris clair et décomposition de P entre secteurs pour le noir. Note de lecture : L'indice d'exposition aux élèves très favorisés était proche de 0,16 en 2004. II peut être décomposé en une part de cette exposition due à des différences intersectorielles (public hors ZEP/privé/ZEP) et une part due à des différences au sein de chaque secteur.

Source : Base Centrale de Scolarité (2004-2014) DEPP - calcul des auteurs.

\subsection{La place relative de chaque secteur tout au long de la distribution des collèges}

Nous analysons à présent la distribution de l'entropie des différents secteurs de l'éducation secondaire au niveau du collège. Pour chaque secteur, les collèges sont donc classés en fonction de leur entropie.

La figure 3 décompose ce classement en percentiles. Les collèges les plus mixtes sont ceux situés à droite de la figure ; la structure sociale des élèves de ces collèges est parmi les plus proches de la distribution sociale avec équi-répartition. Les collèges situés à gauche sont les collèges les moins mixtes. 
Figure 3. Part des collèges selon leur secteur en fonction de leur niveau d'entropie sur la période 2004-2014

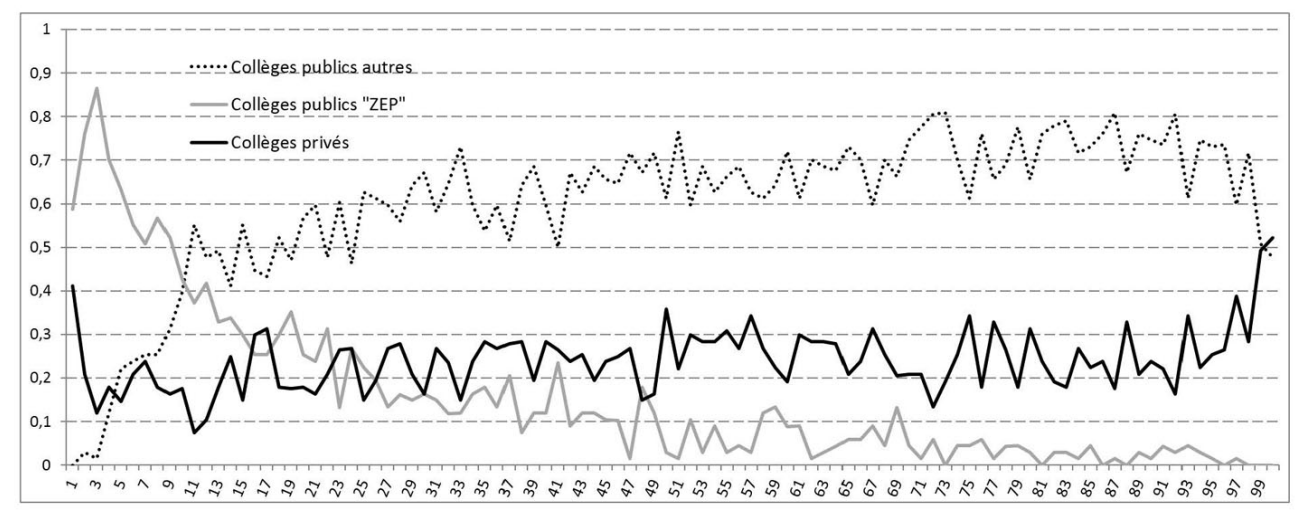

Note de lecture : Parmi les collèges les moins mixtes (1er percentile de niveau d'entropie), la proportion d'élèves en ZEP est proche de $60 \%$. Source : Base Centrales de Scolarité (2004-2014) DEPP - calcul des auteurs.

Sans surprise, les collèges publics en ZEP sont plus homogènes socialement ; ils sont surreprésentés au sein des collèges avec les niveaux d'entropie les plus bas. Ainsi, alors qu'ils représentent près de $16 \%$ des établissements de notre échantillon, leur part est supérieure à $20 \%$ dans le premier quart de la distribution (figure 3). On note par ailleurs que ce type de collège est faiblement représenté au sein des 25 premiers percentiles de collèges les plus mélangés : leur part ne franchit pas les $7 \%$. Leur part relative croît de manière modérée entre le 65 ème et le 25 ème percentile, avant de connaître une forte profession entre le 25 ème percentile et le début de la distribution.

Les collèges privés, qui ne représentent que $24 \%$ des collèges, sont surreprésentés aux deux extrêmes de la distribution soulignant que ce secteur comprend à la fois des collèges plus mixtes et plus "ségrégés " que les autres.

Le secteur public hors éducation prioritaire représente $60 \%$ des établissements. Comme le secteur privé, du fait de l'absence d'établissements en éducation prioritaire au sein des établissements les plus mélangés socialement, le secteur public hors éducation prioritaire est également surreprésenté au sein des établissements les plus mélangés. Cette surreprésentation disparait en deçà du $20^{\text {ème }}$ percentile, c'est-à-dire pour les $20 \%$ d'établissements les plus "ségrégés " de notre échantillon. Ces résultats sont stables pour les différentes années.

Au final, cette décomposition permet de comprendre l'origine de la forte hétérogénéité des collèges privés, soulignée dans le commentaire de la figure 1 et identifiée par Givord $\&$ al. (2016b). En effet, ces collèges sont présents tout au long de la distribution et surreprésentés aux deux extrêmes. Le secteur public parait plus homogène, car les collèges de ce secteur sont moins présents aux extrêmes. 
Deux faits stylisés importants ont été mis au jour par cette analyse. Tout d'abord, en termes de mixité sociale, le secteur public ne peut pas être analysé comme un seul bloc, tant les collèges relevant de l'éducation prioritaire sont concentrés au sein des établissements les moins mélangés socialement. Ensuite, les collèges privés ne se ressemblent pas en termes de mixité sociale ; cette hétérogénéité est fortement masquée lorsque l’on raisonne, par exemple, sur la base de moyennes sectorielles nationales.

Il convient maintenant de vérifier si la manière dont cette mixité sociale opère permet aux élèves très favorisés de préserver une forme "d'entre soi » et contraint les élèves les plus défavorisés à rester dans des collèges ségrégés.

\subsection{Comment les collèges font-ils de la mixité sociale ?}

Partant de la figure 3 de la partie précédente, qui décompose la répartition des collèges par secteur en fonction de leur niveau d'entropie, nous complétons l'analyse en regroupant les collèges en fonction de leurs niveaux d'entropie. L'idée est de comparer les collèges les moins mixtes, les collèges les plus mixtes et un ensemble de collèges médians. Il s'agit de voir si, au sein de chacun de ces groupes, les élèves les plus favorisés se répartissent de manière uniforme ou s'ils se regroupent au sein de certains collèges. Pour chaque groupe, nous distinguons les secteurs public, privé et ZEP. Ces regroupements sont présentés pour les années de début et de fin de la période analysée. Ces groupes reflètent mécaniquement la répartition entre secteur présentée dans la figure 3.

Pour privilégier une présentation simple de nos résultats, nous comparons trois groupes de collèges : ceux du dernier décile d'entropie (groupe A), ceux situés autour du niveau médian ${ }^{14}$ de contribution à l'indice d'entropie (groupe B) et ceux situés dans le premier décile (groupe $\mathrm{C}$ ).

À titre d'illustration, pour l'année 2014, le groupe A des collèges les plus « mixtes » comprend 673 collèges, dont $35 \%$ dans le privé et seulement $1 \%$ en ZEP ; le groupe B des collèges, avec un niveau de mixité médian, regroupe 1345 collèges, dont $7 \%$ en ZEP et $22 \%$ dans le privé ; le groupe $\mathrm{C}$ des collèges les moins " mixtes » regroupe 739 collèges, dont $55 \%$ de collèges en ZEP et $22 \%$ de collèges privés ${ }^{15}$.

Pour chacun des trois groupes (A, B ou C) et au sein de ces groupes, pour chaque secteur (privé, public ou ZEP), nous calculons la répartition par origine sociale des élèves et l'indice d'exposition. Par construction, chaque groupe a un niveau de mixité sociale (telle que mesurée par l'entropie) comparable et ce, quel que soit le secteur considéré. Par exemple, les établissements privés et les établissements en ZEP situés dans le groupe B ont

14. Plus précisément, nous avons retenu ici les collèges situés entre le 40ème et le 60ème percentile.

15. Le nombre de collège n'est pas le même pour les groupes A et $\mathrm{C}$ car la contribution à l'indice d'entropie normalisée est pondérée par la taille du collège : les collèges du groupe le moins mixte sont en moyenne plus petits. 
des niveaux d'entropie voisins : comparer leur répartition par origine sociale des élèves et leur $P$ permet d'appréhender l'ampleur et la nature de l'hétérogénéité subsistante parmi ces établissements. Les résultats sont présentés dans les tableaux 1 et 2.

Tableau 1. Répartition des différentes catégories sociales en 2004 pour les groupes A (« mixte »), B (« médian ») et C (" ségrégé »)

\begin{tabular}{|c|c|c|c|c|c|}
\hline Groupe & Secteur & $\begin{array}{c}\text { Part de « très } \\
\text { favorisés » }\end{array}$ & $\begin{array}{c}\text { Part de } \\
\text { "favorisés " }\end{array}$ & $\begin{array}{c}\text { Part } \\
\text { d' « intermédiaires» }\end{array}$ & $\begin{array}{c}\text { Part de } \\
\text { " défavorisés " }\end{array}$ \\
\hline \multirow[t]{3}{*}{ A } & 1-Public ZEP & $23 \%$ & $12 \%$ & $31 \%$ & $34 \%$ \\
\hline & 2-Public autre & $25 \%$ & $14 \%$ & $33 \%$ & $28 \%$ \\
\hline & 3-Privé & $25 \%$ & $16 \%$ & $35 \%$ & $24 \%$ \\
\hline \multirow[t]{3}{*}{ B } & 1-Public ZEP & $12 \%$ & $8 \%$ & $34 \%$ & $46 \%$ \\
\hline & 2-Public autre & $17 \%$ & $7 \%$ & $36 \%$ & $40 \%$ \\
\hline & 3-Privé & $25 \%$ & $7 \%$ & $42 \%$ & $26 \%$ \\
\hline \multirow[t]{3}{*}{ C } & 1-Public ZEP & $3 \%$ & $3 \%$ & $18 \%$ & $76 \%$ \\
\hline & 2-Public autre & $15 \%$ & $4 \%$ & $22 \%$ & $60 \%$ \\
\hline & 3-Privé & $62 \%$ & $4 \%$ & $16 \%$ & $19 \%$ \\
\hline
\end{tabular}

Note de lecture : dans le groupe A, en 2004, au sein des collèges en ZEP (zone d'éducation prioritaire), la part de collégiens très favorisés est de $23 \%$. Source : Base Centrale de Scolarité (2004-2014) DEPP - calcul des auteurs.

Tableau 2. Répartition des différentes catégories sociales en 2014 pour les groupes A (« mixte »), B (« médian ») et C (« ségrégé »)

\begin{tabular}{|c|c|c|c|c|c|}
\hline Groupe & Secteur & $\begin{array}{c}\text { Part de «très } \\
\text { favorisés » }\end{array}$ & $\begin{array}{c}\text { Part de } \\
\text { "favorisés " }\end{array}$ & $\begin{array}{c}\text { Part } \\
\text { d' « intermédiaires » }\end{array}$ & $\begin{array}{c}\text { Part de } \\
\text { " défavorisés " }\end{array}$ \\
\hline \multirow[t]{3}{*}{ A } & 1-Public ZEP & $20 \%$ & $10 \%$ & $33 \%$ & $36 \%$ \\
\hline & 2-Public autre & $26 \%$ & $10 \%$ & $34 \%$ & $30 \%$ \\
\hline & 3-Privé & $28 \%$ & $13 \%$ & $37 \%$ & $22 \%$ \\
\hline \multirow[t]{3}{*}{ B } & 1-Public ZEP & $14 \%$ & $5 \%$ & $34 \%$ & $46 \%$ \\
\hline & 2-Public autre & $19 \%$ & $5 \%$ & $37 \%$ & $40 \%$ \\
\hline & 3-Privé & $30 \%$ & $6 \%$ & $41 \%$ & $23 \%$ \\
\hline \multirow[t]{3}{*}{ C } & 1-Public ZEP & $3 \%$ & $2 \%$ & $20 \%$ & $75 \%$ \\
\hline & 2-Public autre & $12 \%$ & $3 \%$ & $23 \%$ & $63 \%$ \\
\hline & 3-Privé & $70 \%$ & $3 \%$ & $17 \%$ & $10 \%$ \\
\hline
\end{tabular}

Note de lecture : dans le groupe A en 2014, au sein des collèges en ZEP (zone d'éducation prioritaire), la part de collégiens très favorisés est de $20 \%$. Source : Base Centrale de Scolarité (2004-2014) DEPP - calcul des auteurs.

Le groupe A (ou groupe « mixte ») réunit l'ensemble des établissements où la mixité sociale est la plus élevée ${ }^{16}$. En dépit de cette homogénéité des niveaux d'entropie, nous constatons que les répartitions d'élèves par origine sociale sont loin d'être identiques selon les secteurs. En 2004, la part d'élèves « très favorisés » dans le privé est proche de $25 \%$. C'est,

16. Comme expliqué dans la sous-partie 2.2, les établissements privés y sont donc relativement nombreux et les collèges ZEP plutôt rares. 
certes, inférieur à la proportion observée dans l'ensemble du privé (29\% en 2004), mais reste comparable aux proportions observées dans les collèges publics hors ZEP (25\%) du groupe A et supérieur à la proportion des collèges en ZEP de ce même groupe (23\%). Les parts d'élèves " favorisés " ou " moyens " en collèges privés " mixtes " (i.e., du groupe A) sont aussi supérieures à celles observées dans le public, ZEP ou non, "mixte ». En conséquence, la part d'élèves "défavorisés " y est nettement plus faible qu'en ZEP ou dans le secteur public hors ZEP. Cette analyse révèle qu'il existe différentes façons de " produire » de la mixité, et celle du privé diffère de celle du public. Les collèges privés les plus mixtes concentrent des proportions relativement importantes d'élèves allant de " très favorisés » à " moyens ». Les catégories sociales défavorisées y restent sous-représentées en comparaison d'autres secteurs, même si leur part y est supérieure à celle observée dans l'ensemble du secteur privé. De manière similaire, la tendance des ZEP à accueillir une proportion plus importante d'élèves « défavorisés » que les autres collèges est toujours vraie, même si l'écart est moindre, au sein du groupe des collèges avec la plus forte mixité sociale. Ce tropisme vers les catégories " défavorisées ", pour les ZEP mixtes, s’est amplifié avec le temps. En 2014, la part d'élèves "défavorisés » en ZEP du groupe A est de $36 \%$ (contre $34 \%$ en 2004). Dans le même temps, la part d'élèves défavorisés dans le privé du groupe A a diminué entre 2004 et 2014, passant de $24 \%$ à $22 \%$.

Figure 4. Indices d'Exposition Normalisés $P$ par groupe, secteur et année. Catégorie de référence : élèves très favorisés

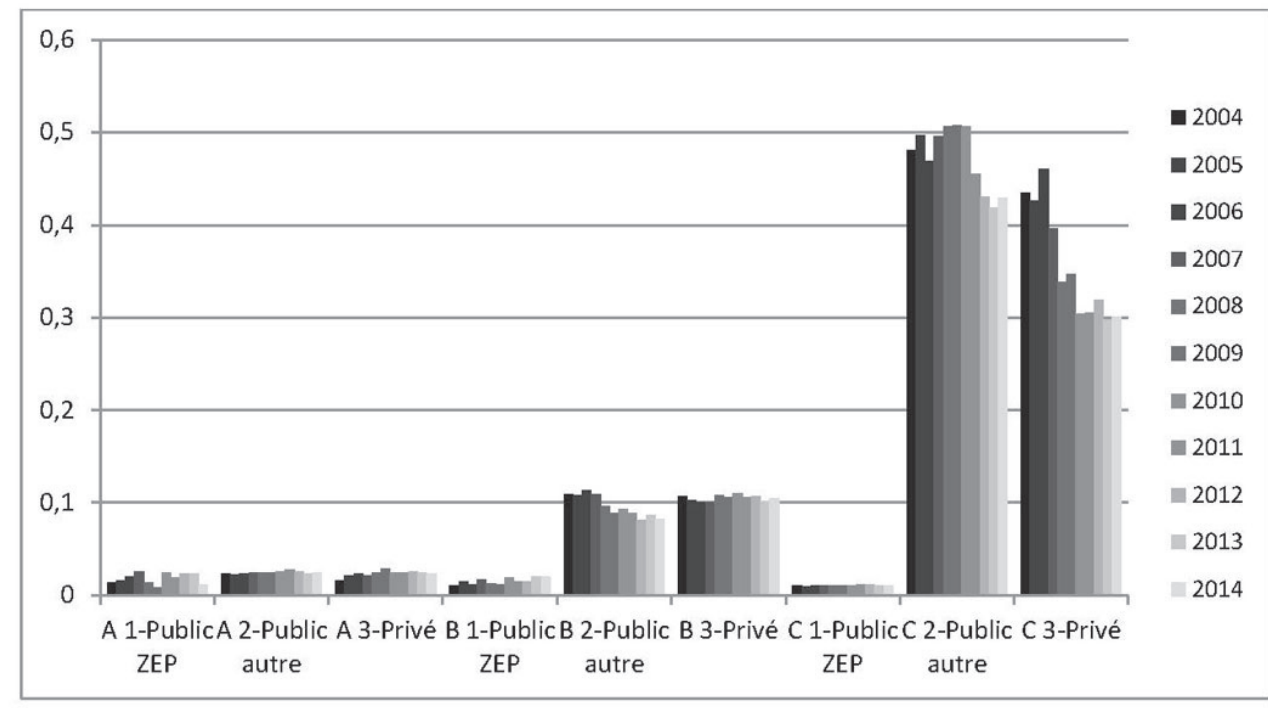

Note de lecture : Le groupe A (groupe « mixte ») correspond aux niveaux d'entropie les plus élevés ; le groupe B (" groupe médian ») à des niveaux d'entropie proches de la médiane et le groupe C (groupe « ségrégé ») à des niveaux d'entropie très faibles. Dans le groupe C, au sein des établissements privés, l'indice d'exposition était d'environ 0,3 en 2014.

Source : Base Centrales de Scolarité (2004-2014) DEPP - calcul des auteurs. 
La figure 4 montre les indices d'exposition normalisés (référence : élèves très favorisés) pour chacun des secteurs (public non ZEP, ZEP et privé) au sein de chacun des groupes (A, B et $\mathrm{C}$ ), par année, indépendamment de l'effet moyen présenté dans le tableau 1. L'analyse de cet indice nous permet d'insister sur les différences qu'il existe entre les collèges d'un même secteur et avec un niveau comparable de mixité sociale. Cela permet d'évaluer si une hétérogénéité sociale subsiste entre collèges d'un même secteur regroupés par niveau d'entropie. Quel que soit le secteur considéré, les indices d'exposition normalisés sont extrêmement faibles dans le groupe A. Cela signifie que les répartitions par origine sociale, fournies dans les tableaux $\mathbf{1}$ et $\mathbf{2}$, sont relativement uniformes au sein de chaque secteur. Peu d'hétérogénéité intra-secteur subsiste au sein du groupe A. Au final, les collèges " mixtes » se ressemblent au sein de chaque secteur.

Pour le groupe B (ou groupe "médian »), les tendances déjà mises en évidence pour le groupe A se sont amplifiées. Les collèges privés " médians " ont une proportion relativement élevée d'élèves " très favorisés » et les collèges en ZEP " médians " ont une proportion relativement élevée d'élèves "défavorisés ». Ces résultats obtenus pour l'année 2004 sont toujours qualitativement valables en 2014.

À côté de ce résultat logique, compte tenu de la surreprésentation globale des élèves favorisées dans le privé, l'analyse de l'indice d'exposition fait apparaître un élément nouveau : au sein du groupe $\mathrm{B}$, la ségrégation dans l'exposition au groupe social "très favorisé " est plus élevée dans le privé et dans le public hors ZEP qu'en ZEP. Autrement dit, les établissements en ZEP du groupe $B$ restent assez homogènes. Les élèves «très favorisés " y sont relativement peu nombreux, mais répartis de manière relativement uniforme. Au contraire, les élèves "très favorisés " hors ZEP (public ou privé) sont relativement nombreux et répartis de manière inégale. Alors même que tous les établissements du groupe B sont comparables en termes de mixité sociale, on observe une hétérogénéité intra-sectorielle dans le public hors ZEP et dans le privé, mais pas en ZEP. Sur la période, la figure 4 indique que «l'entre soi » des très favorisés au sein des collèges médians en termes de mixité sociale a légèrement reculé dans le public hors ZEP et est resté stable dans le privé.

Ces résultats se trouvent accentués en analysant le groupe $\mathrm{C}$ (ou groupe "ségrégé »). En observant les répartitions des origines sociales des élèves par secteur, on pourrait être tenté de croire qu'il y a deux modèles : celui des ZEP (et dans une moindre mesure le secteur public hors ZEP) où la proportion d'élèves "défavorisés » est très élevée (près de $76 \%$ en 2004, $60 \%$ dans le public hors ZEP) et celui du secteur privé où la proportion d'élèves "très favorisés " est très forte (près de $62 \%$ ). Chacun de ces secteurs ségrége "à sa façon ", avec une proportion extrême de "très favorisés " ou de "défavorisés ".

Cependant, l'indice d'exposition révèle que le constat doit être plus nuancé puisque la ségrégation intra-ZEP est quasi-nulle, alors qu'elle est très forte dans les autres secteurs. Cela signifie qu'en ZEP, dans le groupe " ségrégé ", les élèves issus de milieux " très favorisés " sont rares, mais uniformément répartis. Dans le privé, ce sont les élèves 
" défavorisés " qui sont rares, mais ils côtoient peu d'élèves très favorisés dans leur collège : une forte hétérogénéité intra-sectorielle subsiste, hors ZEP. Parmi les collèges privés fortement ségrégés cohabitent différents types de modèles : ceux, majoritaires, avec une proportion très importante d'élèves " très favorisés " (plus encore que dans la répartition moyenne du groupe $\mathrm{C}$ ) et ceux, minoritaires, où la proportion d'autres origines sociales est importante. Enfin, en dépit de la proportion relativement faible d'élèves très favorisés dans le public hors ZEP du groupe $\mathrm{C}$, les niveaux de ségrégation y sont très élevés (plus encore que dans le privé). Sur la période, la figure $\mathbf{4}$ montre que la tendance à la préservation de "l'entre soi " des très favorisés est devenue moins forte dans le public et dans le privé ; cependant cette baisse est beaucoup plus marquée dans le privé. Au sein du secteur public, cela suggère l'existence et le maintien, tout au long de la période, d'un nombre restreint d'établissements " élitistes ", regroupant quasi-exclusivement des élèves très favorisés.

\section{Conclusion}

Les politiques visant à favoriser la mixité sociale à l'école devraient s'appuyer de manière plus systématique sur des outils de mesure de la ségrégation. En effet, nous mettons ici en évidence une hétérogénéité au sein des secteurs privés, en ZEP et hors ZEP. Dans cet article, l'utilisation combinée de deux types d'indices de ségrégation (entropie et exposition) nous a permis de dégager un ensemble de faits stylisés sur la mixité sociale des collèges en France, selon leur secteur (public, privé et éducation prioritaire) durant la période 2004-2014.

Tout d'abord, il apparaît que le secteur privé est légèrement surreprésenté parmi les collèges les plus mixtes et au sein des collèges les moins mixtes, c'est-à-dire aux extrêmes de la distribution des niveaux de mixité sociale (entropie). La mise en évidence de ce fait stylisé constitue bien un apport de ce travail à la littérature sur le sujet de la mixité sociale au collège (cf. introduction). A contrario, dans le secteur public, c'est bien le secteur de l'éducation prioritaire qui concentre les collèges les moins mixtes et le secteur hors éducation prioritaire qui concentre les collèges les plus mixtes

Ensuite, il n'est pas avéré que les collèges du public et du privé « ségrégent " chacun à leur façon (c'est-à-dire en concentrant les élèves très favorisés pour le privé et les élèves défavorisés pour le public) ${ }^{17}$. L'analyse de la mixité sociale ne peut se contenter de cette opposition trop frustre pour rendre compte à un niveau national de la diversité des pratiques. En effet, nous montrons que parmi les collèges les plus mixtes, les collèges du secteur privé, même s'ils ont plus d'élèves défavorisés que les autres collèges privés, recrutent moins d'élèves de ce type que leurs homologues mixtes du public ce qui confirme des résultats déjà mis en avant par la littérature sur le sujet. Cependant,

17. Pour cela, nous avons examiné la manière dont se répartissent les élèves très favorisés au sein de trois groupes de collèges différenciés (mixtes, médians et ségrégés), sur la base de leur degré de mixité sociale. 
au sein de ces collèges les plus mixtes, les élèves très favorisés sont répartis de manière homogène entre les établissements, sans différence sectorielle (privé/public ou éducation prioritaire). Dans ces collèges, il n'y a donc pas de préservation d'une forme "d'entre soi » des élèves très favorisés, ni dans le public, ni dans le privé.

Nos résultats révèlent également, parmi les collèges les moins mixtes, une forte différenciation entre les collèges publics en ZEP, d'une part, et les autres collèges, d'autre part. Les élèves très favorisés sont uniformément répartis dans les collèges en ZEP. En revanche, ce n'est pas le cas dans le reste du secteur public ni dans le privé.

Nous mettons en évidence une tendance à la baisse de "l'entre soi " des élèves très favorisés dans le privé. Cette baisse est beaucoup moins forte pour les collèges publics hors ZEP appartenant à ce groupe ; l'entre soi des très favorisés y a beaucoup moins diminué sur la période et reste à un niveau plus élevé que celui observé dans le secteur privé. Là encore, la mise en évidence de ce fait stylisé constitue un apport de ce travail à la littérature sur le sujet.

Nous obtenons des résultats similaires, mais beaucoup moins marqués, pour le groupe de collèges médians en termes de mixité. Ainsi, le niveau d'entre soi des très favorisés est du même ordre dans le public hors ZEP et dans le privé, mais à un niveau beaucoup moins important que dans le cas des collèges les moins mixtes; alors que les élèves très favorisés ont tendance à être répartis de manière relativement uniforme au sein des ZEP.

Faire progresser la mixité sociale nécessite certainement de cibler un certain nombre d'actions sur les collèges publics relevant de l'éducation prioritaire (ZEP), mais au final, nos résultats montrent que l'opposition public/privé apparaît peu pertinente pour structurer des politiques publiques en faveur de la mixité sociale.

En effet, cette opposition ne permet pas de rendre compte des différents modèles de mixité (et d'absence de mixité) présents au sein de chaque secteur, notamment car le privé est très hétérogène et croise plusieurs dimensions territoriales très diverses — l'enseignement privé catholique est historiquement bien implanté dans certaines régions, les grandes villes connaissent également un secteur privé plus segmenté socialement (cf. Poucet, 2012).

De ce point de vue, analyser plus avant la dimension territoriale et l'environnement dans lequel opèrent les différents collèges (notamment au-delà du "modèle de mixité " d'un collège donné, intégrer les "modèles de mixité » des établissements les plus proches géographiquement) constitue une voie de recherche intéressante permettant de réarticuler les politiques favorisant le développement de la mixité sociale à l'école avec les politiques au niveau territorial. 


\section{Bibliographie}

Audren G., Lorcerie F. (2013), «À Marseille : une école polarisée mais diverse », Revue Projet, 2013/2, 333, pp. 42-51.

Base centrale de scolarité (BCS) (2004 et 2005 DEP), (2006-2014 DEPP), ministère de l'Éducation nationale [producteur], ADISP-CMH [distributeur].

Collectif Coysevox (2016), "Quand les institutions jouent les pompiers pyromanes. La mixité sociale dans les collèges ", Metropolitiques, www.metropolitiques.eu.

Courtioux P. (2016), "Dix ans de mixité sociale au collège : le public fait-il vraiment mieux que le privé ? ", Position Paper EDHEC, Mai.

Durand Y., Salles R. (ed.) (2015), Rapport d'information sur l'évaluation des politiques publiques en faveur de la mixité sociale dans l'éducation nationale, Assemblée nationale, $\mathrm{n}^{\circ} 3292$.

Fack G., Grenet J. (2009), "Sectorisation des collèges et prix du logement à Paris ", Actes de la recherche en Science sociales, 180(5), pp. 44-62.

Frankel D.,Volij O. (2011), "Measuring school segregation”, Journal of Economic Theory, 146 (1), pp. $1-38$

Givord P., Guillerm M., Monso O., Murat F. (2016a) «Comment mesurer la ségrégation dans le système éducatif ? Une étude de la composition sociale des collèges français ", Education \& Formations, 91, pp. 21-51.

Givord P., Guillerm M., Monso O., Murat F. (2016b) « La ségrégation sociale entre les collèges. Quelles différences entre public et privé, aux niveaux national, académique et local ?», Education \& Formations, 91, pp .53-76.

James D., Taeuber K. (1985), "Measures of segregation”, Sociological methodology, 14, pp. 1-32.

Ly S.-T., Maurin E., Riegert A. (2014), La mixité sociale et scolaire en Ile-de-France : le rôle des établissements, Rapport de l'Institut des Politiques Publiques, $\mathrm{n}^{\circ} 4$.

Ly S.-T., Riegert A. (2014), " Mixité sociale et scolaire et ségrégation inter et intra établissement dans les collèges et lycées français ", Conférence nationale d'évaluation des systèmes scolaires, juin.

Maguain D. (2009), "La suppression de la sectorisation est-elle une bonne chose ?", Revue d'économie politique, 119 (4), pp. 569-612.

Massey D., Denton N. (1988), “The dimensions of residential segregation”, Social Forces, 67 (2), pp. 281-235. 
Merle P. (2010), « Structure et dynamique de la ségrégation sociale dans les collèges parisiens ", Revue française de pédagogie, 170, pp. 73-85.

Merle P. (2012), La ségrégation scolaire, Paris, La Découverte, Repère.

OCDE (2016), Résultats du PISA 2015 (Volume I) : L'excellence et l'équité dans l'éducation, Paris, Éditions OCD.

Poucet B. (2012), L'enseignement privé en France, Que-sais-je ?, Paris, Presses Universitaires de France.

Prost A. (2013), «L'enseignement privé prisonnier de son héritage », Revue Projet, 2013/2, 333, p. 33-41

Radica K. (1995), "Taux plafonds d'élèves en zones d'éducation prioritaires », document DEPP.

Reardon S, Firebaugh G. (2002), “Measures of multigroup segregation”, Sociological methodology, 32 (1), pp. 33-67.

Reardon S., O’Sullivan D. (2004), "Measures of spatial segregation”, Sociological methodology, 34 (1), pp. 121-162.

Thélot C. (2013), «Les missions de l'école en France, contribution de l'école catholique », Revue Projet, 2013/2, 333, pp. 4-12.

Trancart D. (2012), "Quel impact des ségrégations socio-spatiales sur la réussite au collège ", Formation Emploi, 120, pp. 35-55.

Thaurel-Richard M., Murat F. (2013), «Evolution des caractéristiques des collèges durant la mise en œuvre de l'assouplissement de la carte scolaire en 2007 ", Education et Formation, n' 83 , pp. 11-23.

Van Zanten A. (2009), Choisir son école, Paris, Presses Universitaires de France.

Van Zanten A., Obin J.-P. (2008), La carte scolaire, Paris, Presses Universitaires de France, "Que-sais-je?".

Van Zanten A., da Costa S. (2013), «La gestion de la carte scolaire dans la périphérie parisienne. Enjeux, dynamiques et limites de la gouvernance éducative locale ", Education et Formation, n ${ }^{\circ} 83$, pp. 99-107. 